\title{
Knowledge-based Auto Repair Diagnosis System and Application
}

\author{
Fang Xiaofen \\ School of Mechanical and Electrical Engineering \\ Quzhou College of Technology \\ Quzhou, China \\ e-mail: fangxiaofen1985@hotmail.com \\ Xu Jianliang \\ School of Mechanical and Electrical Engineering \\ Quzhou College of Technology \\ Quzhou, China \\ e-mail: xj121@qq.com
}

\author{
Fang Kunli \\ School of Mechanical and Electrical Engineering \\ Quzhou College of Technology \\ Quzhou, China \\ e-mail: 278857958@qq.com \\ Yang Fan \\ School of Mechanical and Electrical Engineering \\ Quzhou College of Technology \\ Quzhou, China \\ e-mail: 350577218@qq.com
}

\begin{abstract}
Auto repair is a process which solves the problem iteratively and constantly. It is required to establish the knowledge base model and the inference base model. Meanwhile, it develops the knowledge-based auto repair diagnostic system through the integration of the auto repair experience and the repair process. Afterwards, the repair time can be effectively shortened and the repair complexity can be reduced. Finally, the rapid repair and the efficient service will be achieved.
\end{abstract}

Keywords-knowledge management; Expert system; artificial Intelligence; repair diagnostic system

\section{INTRODUCTION}

With the rapid development of Chinese auto industry, the categories of the brand of auto repair is increasing, so as the the difficulty of diagnosis and repair has been increased. For the automotive after-sales service, the quality and the efficiency of auto repair are the main factors that would affect the customer satisfaction[1-2]. Accordingly, it is crucial to improve the quality and the efficiency of repair in the process of auto repair and management. Therefore, an expert query system that can guide the auto repair is needed [3-6]. The repair data, the standards, the equipment, the tools, the technology requirements and the operating process can be sent to the staffs in the repair process through the knowledge-based auto repair and diagnostic system.

The auto fault diagnosis system can be categorized into two parts: the first one is developed for a component or the subsystem of cars (i.e. The direct injection engine and the automatic transmission can achieve the rapid repair and diagnostic through the identify of corresponding fault codes); the second one refers that the repair information can be obtained from the inference method through the troubleshooting flowchart in order to guide the maintenance and the production. Only the faults involved in the flow chart can be recognized, which is different with the real data; such that this method is not recommended.

The knowledge-based repair diagnostic system can collect all possible repair diagnostic information and expert experience so that it is able to support all the stages included [7].

The development of this system is based on the knowledge, the database and the web technology. Meanwhile, the automotive fault tree is used to establish the dynamic knowledge database and the inference database. For the auto repair work, this system would collect real time data to give guidance. The maintenance staff could query and use the system with a significantly higher efficiency.

\section{MODEL OF KNOWLEDGE-BASED AUTO REPAIR DIAGNOSTIC SYSTEM}

\section{A. Knowledge-based Expert System}

The Expert System is a computer program, which is able to solve some problems in certain fields as experts. The expert system generally separates the knowledge of the application field to compose the entity of a knowledge base. The processing of the knowledge base is conducted by the identification control strategy that is independent of the knowledge base. The repair expert system has a huge knowledge base. It consists of levels of data, the knowledge base and the control library. The knowledge base stores the knowledge about the automotive fault diagnosis. In addition, the system control level usually expresses the inference rules. The general structure of expert system refers to the system which takes a MYCIN system as the representative and that is based on the rules [8-9], including the knowledge base, the inference engine, the comprehensive database, the manmachine interface, the interpreter and the access procedures of knowledge base. The knowledge-based auto repair diagnostic system employs this kind of structure. It is required to establish the dynamic knowledge base and the inference base according to the diagnosis inference rules of auto fault tree. The web technology is adopted to establish the database engine and the good human-computer interface. The maintenance staffs can easily and quickly conduct the repair work under the computer guidelines. 


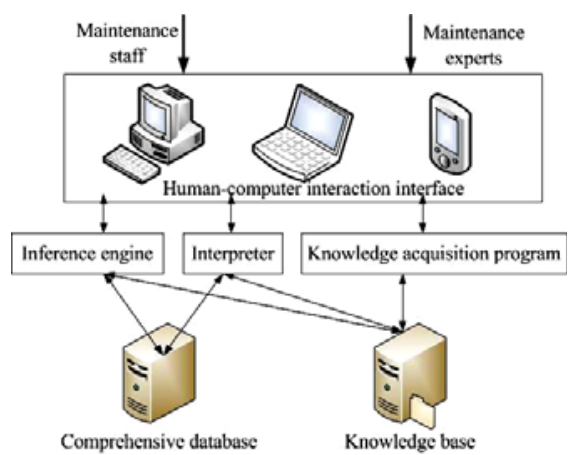

Figure 1. Model of auto repair expert system based on rules

\section{B. Knowledge Representation of Repair Diagnostic System}

When establishing the fault diagnosis expert system, they should be classified according to the fault characteristics. Each category should be subdivided as much as possible. Afterwards, it is necessary to create the auto troubleshooting flowchart. The task of knowledge representation is to transform the mutually independent or associated fault trees to the forms that computers can recognize, store and query. Then, the knowledge nodes should be coded and avoid the conflicts between the knowledge as much as possible. The smooth flow from root to leaf should be ensured.

Fault tree of auto repair

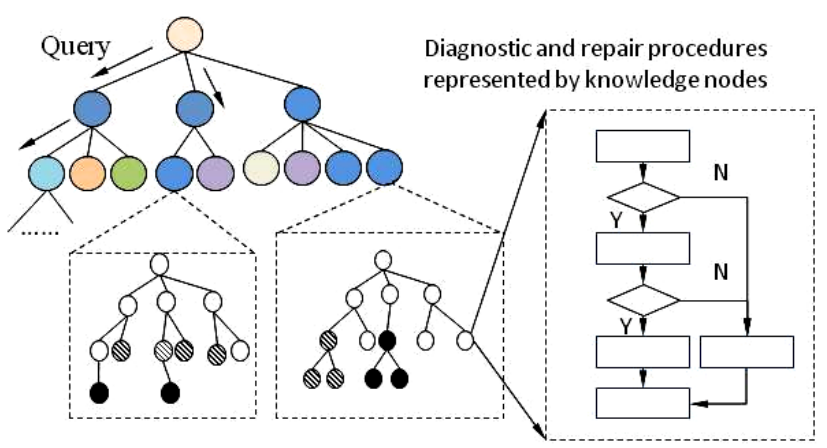

$$
\begin{aligned}
& \text { Suspicious fault } \\
& \text { node of car }
\end{aligned}
$$

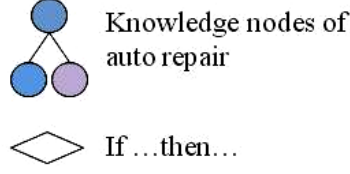

Figure 2. Levels of fault tree of auto diagnosis and repair

The repair knowledge nodes are defined as:

$$
\mathrm{K}_{\mathrm{N}}=\left\{\mathrm{K}_{\Lambda}, \mathrm{K}_{\mathrm{T}}, \mathrm{K}_{\mathrm{o}}, \mathrm{K}_{\chi}\right\}
$$

$\mathrm{K}_{\Lambda}$ is the knowledge level, $=\{$ parent node, child node, similar node $\}. \mathrm{K}_{\mathrm{T}}$ is the type of knowledge, $\mathrm{K}_{\mathrm{T}}$ $=\{$ process knowledge, conceptual knowledge, selective rule knowledge $\} \quad \quad \mathrm{K}_{\mathrm{o}}$ is the encoding of knowledge nodes, $\mathrm{K}_{0}=$ sstored knowledge node encoding, corresponding encoding of process node $\}. \mathrm{K}_{\chi}$ is the knowledge node with potential conflict, $\mathrm{K}_{\chi}=\{$ parent node, child node, similar node\}.

We defined each knowledge node as one class. In the knowledge storage system of automobile fault diagnosis tree, Web Ontology Language (OWL) [10-13] is used to represent the structure of knowledge. The knowledge base consists of several OWL documents. As XML documents, OWL documents are tree type documents. Each node of the tree is an XML element. The relationships between elements are parent-child or sibling relationships. That is because each OWL document can be represented as a state space of a tree structure.

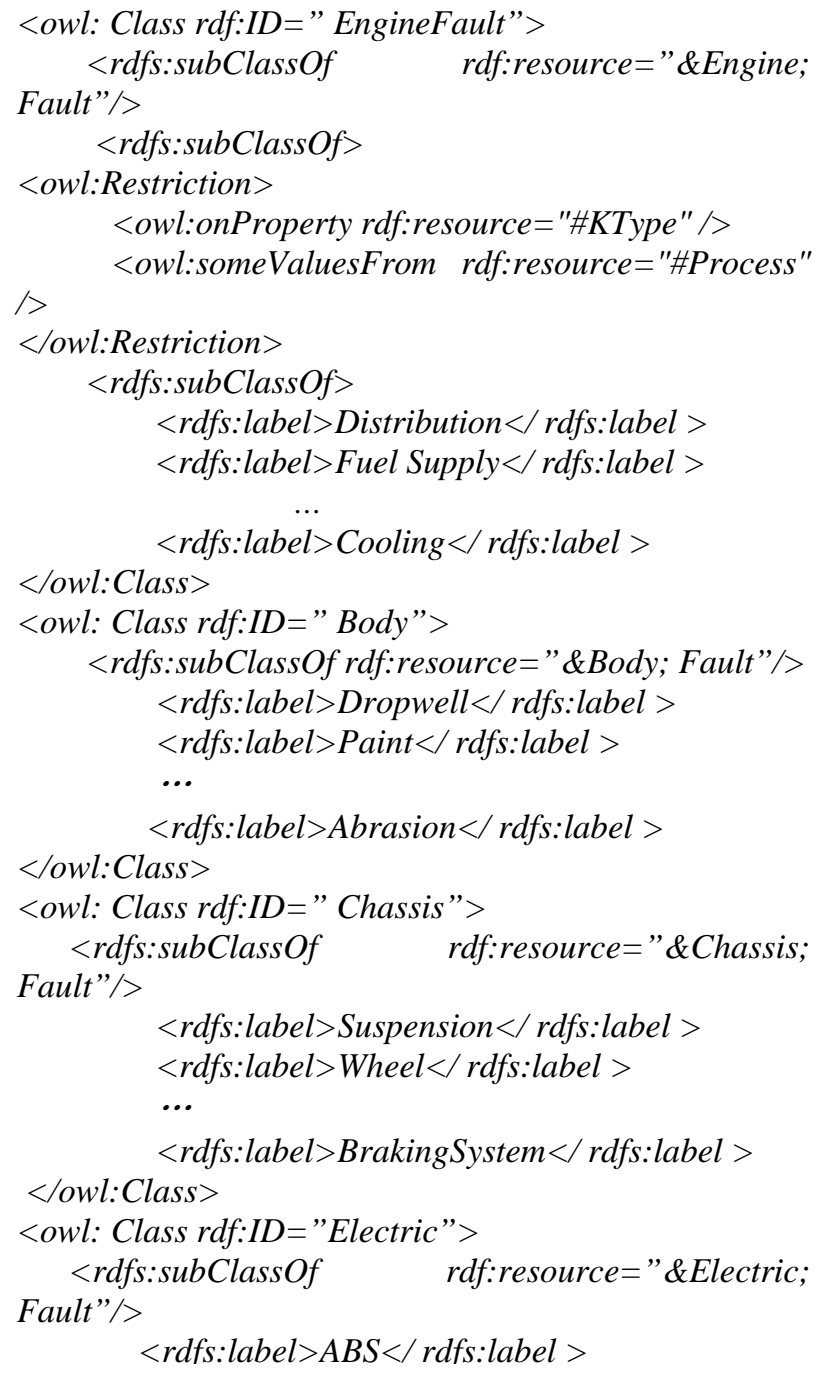

The relationship between two knowledge nodes is defined as a $k_{i j}$. For example, we have $n$ knowledge nodes, the relationship can be expressed as a matrix $R$. 


$$
R=\left[\begin{array}{cccc}
k_{11} & k_{12} & \cdots & k_{1 n} \\
k_{21} & k_{22} & \cdots & k_{2 n} \\
\cdots & \cdots & \cdots & \cdots \\
k_{n 1} & k_{n 2} & \cdots & k_{n n}
\end{array}\right]
$$

Strong coupling state between two knowledge nodes indicates the presence of strong relationship between the two knowledge nodes. Correspondingly, if the two nodes have medium degree of coupling, that relationship between the two knowledge nodes would be medium. If weak coupling exists with two nodes, show two knowledge nodes for the weak relationship.

\section{Knowledge Representation of Repair Diagnostic System}

When establishing the fault diagnosis expert system, it is required to classify them according to the fault characteristics. Each category should be subdivided as much as possible. Afterwards, it is necessary to create the auto troubleshooting flowchart. The task of knowledge representation is to make the mutually independent or associated fault trees become the forms that the computer can recognize, store and query. Then, the knowledge nodes should be coded and it is required to avoid the cross and the conflict between the knowledge as much as possible. The smooth flow from root to leaf should be ensured.

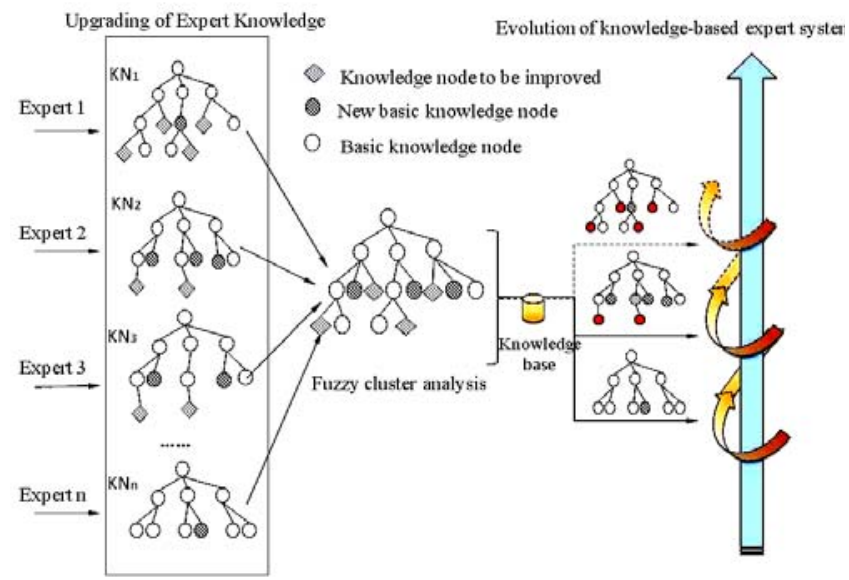

Figure 3. Levels of fault tress of auto diagnosis and repair

When the repair staff upgrades the tacit knowledge into the system, we need to add a new node or improve existed nodes content in the system. With the constantly upgrade and evolution of the knowledge database, the automobile fault diagnosis system will be more and more effective to guide the vehicle repair process based on knowledge.

\section{APPLICATION OF KNOWLEDGE-BASED AUTO REPAIR DIAGNOSTIC SYSTEM}

When the maintenance staff selects the appropriate knowledge module to repair the cars, the sent knowledge can be adopted to complete the simple repairs. Moreover, the
CAD software is employed to conduct the visual modeling for automobile structure. J2EE helps to establish the knowledge base to check the human-computer interaction Web interface. MySQL is considered as the database of structured knowledge.

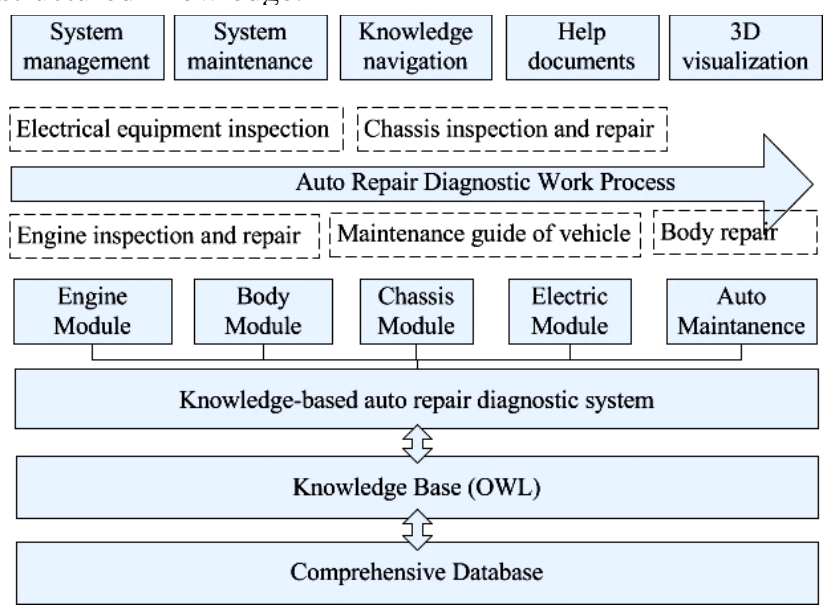

Figure 4. Module structure of the knowledge-based auto repair diagnostic system

The car ignition is a common fault in practice. It is required to enter the knowledge-based auto repair diagnostic interface after selecting the appropriate models, as shown in Figure 5. The users can press "Repair Navigate" and diagnose step by step under the guide of system according to the fault location, the phenomenon or the self-defined search. The maintenance experience is updated to the expert system by the maintenance staff in order to expand the repair knowledge base. Along with the upgrading and evolution, the experience can become the basic knowledge node data of system, which makes the auto repair expert system have the self-learning function and the abilities of expansion and updating.

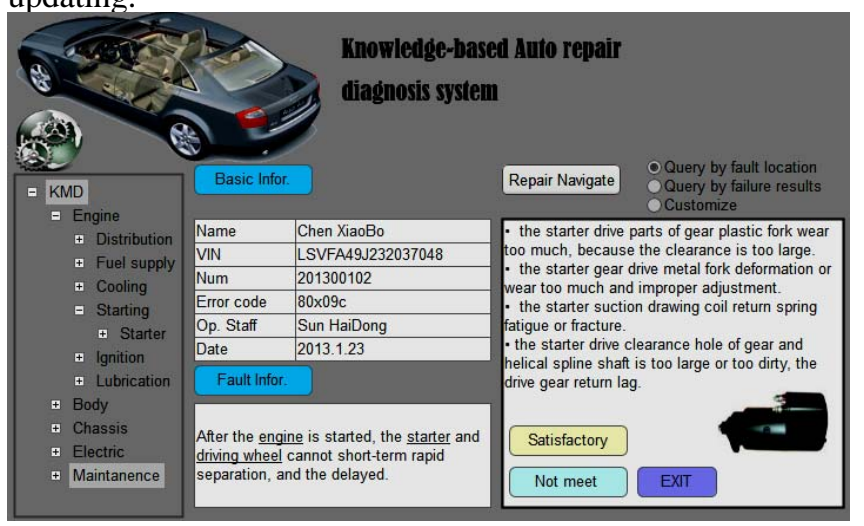

Figure 5. Knowledge-based Auto repair diagnosis systemApplication of knowledge-based auto repair diagnostic system

\section{CONCLUSION}

In conclusion, the Auto repair and diagnosis system based on knowledge innovates in the following aspects: 
- It establishes the processing rules based on the existing database in order to apply the knowledge. The repair efficiency would generate a significant efficiency.

- Knowledge-based vehicle repair diagnostic system is an open system. It has the ability of self-evolution so that the knowledge is constantly updated and improved. Consequently, the function makes the repair more and more convenient.

- The automotive repair service industry involves a large number of disciplines. The effective application of KMS is able to categorize and manage the experience knowledge, in order to enhance the soft power of enterprises.

Future work may include:

- $\quad$ The knowledge may need further categorization to store and to apply the knowledge more efficiency. It could be achieved by the utilization of data mining.

- A method to apply the knowledge based on the repair process may be realized by integrating the process knowledge and process node.

- Ontology may be applied to effectively establish knowledge nodes.

\section{ACKNOWLEDGMENT}

This work was supported by Quzhou Science and Technology Development Fund under Contract No.20111042, Science and Technology Department of Zhejiang province public welfare research project (No. 2013C32SA900004) and Zhejiang Province science and technology plan projects (No.20121055). The authors specially thank Prof. Yangjian Ji for his valuable contribution to this work. F.X thanks Ms Xie for her great support to this work.

\section{REFERENCES}

[1] Valarie A.Zeithaml, Leonard L.Berry, and Parasuraman, A, "The nature and determinants of customer expectations of service," Journal of the Academy of Marketing Science, vol. 12, no. 1, pp. 1-12, 1993.
[2] V.Rohit, "An empirical analysis of management challenges in service factories, service shops, mass services and professional services," Journal of Service Management, vol. 11, no. 1, pp. 8-25, 2000.

[3] J. S. Liang, "A web-based automotive refrigeration troubleshooting system applying knowledge engineering approach,” Computers in Industry, vol. 61, no. 1, pp. 29-43, 2010.

[4] M. Tian, "Fuzzy neural network diagnose expert system of engine." pp. 154-156, 2012 [Intelligent Control and Information Processing (ICICIP), 2012].

[5] B. K. Bose, "Expert system, fuzzy logic, and neural network applications in power electronics and motion control," Proceedings of the IEEE, vol. 82, no. 8, pp. 1303-1323, 1994.

[6] N. Vargas Hernandez, G. Okudan Kremer, L. C. Schmidt, and P. R. Acosta Herrera, "Development of an expert system to aid engineers in the selection of design for environment methods and tools," Expert Systems with Applications, vol. 39, no. 10, pp. 9543-9553, August, 2012.

[7] J. P. Wang, "Analysis in the Mercedes-Benz Automotive Fault Diagnosis,” Applied Mechanics and Materials, vol. 273, pp. 604-608, 2013.

[8] L. Kan, Research on Knowledge Navigation Technology Supporting Complex Product Design, Zhejiang University, Hangzhou, P.R.China, 2011.

[9] P. Li, S.-F. Liu, and Z.-G. Fang, "Interval-valued intuitionistic fuzzy numbers decision-making method based on grey incidence analysis and MYCIN certainty factor," Control and Decision, vol. 27, no. 7, pp. 1009-1014, 2012.

[10] X. H. Wang, D. Q. Zhang, T. Gu, and H. K. Pung, "Ontology based context modeling and reasoning using OWL," Pervasive Computing and Communications Workshops,.Proceedings of the Second IEEE Annual Conference on. IEEE, pp. 18-22, 2004.

[11] S. Bechhofer, F. Van Harmelen, J. Hendler, I. Horrocks, D. L. McGuinness, P. F. Patel-Schneider, and L. A. Stein, "OWL web ontology language reference," W3C recommendation, vol. 10, pp. 2006-01.

[12] B. Motik, U. Sattler, and R. Studer, "Query answering for OWL-DL with rules," Web Semantics: Science, Services and Agents on the World Wide Web, vol. 3, no. 1, pp. 41-60, 2005.

[13] D. Allemang, and J. Hendler, Semantic web for the working ontologist: effective modeling in RDFS and OWL: Morgan Kaufmann,2011. 